\title{
Leitthema
}

Bundesgesundheitsbl 2016 · 59:88-97

DOI 10.1007/s00103-015-2263-x

Online publiziert: 23. Oktober 2015

c) Springer-Verlag Berlin Heidelberg 2015

CrossMark

Albert Nienhaus $^{1,4}$. Claudia Drechsel-Schlund ${ }^{2} \cdot$ Heike Schambortski ${ }^{3}$. Anja Schablon ${ }^{4}$

${ }^{1}$ Abteilung Grundlagen der Prävention und Rehabilitation, Berufsgenossenschaft für Gesundheitsdienst und Wohlfahrtspflege (BGW), Hamburg, Deutschland

${ }^{2}$ BGW, Geschäftsführung der Bezirksverwaltung Würzburg, Würzburg, Deutschland

${ }^{3}$ Abteilung Präventionskoordination, BGW, Würzburg, Deutschland

${ }^{4}$ Institut für Versorgungsforschung in der Dermatologie und bei Pflegeberufen (IVDP), Kompetenzzentrum für Epidemiologie und Versorgungsforschung bei Pflegeberufen (CVcare), Universitätsklinikum Hamburg-Eppendorf, Hamburg, Deutschland

\section{Gewalt und Diskriminierung am Arbeitsplatz}

\section{Gesundheitliche Folgen und settingbezogene Ansätze zur Prävention und Rehabilitation}

Gewalt am Arbeitsplatz ist ein weitverbreitetes Problem, das nicht nur Beschäftigte bei der Polizei oder in der Psychiatrie, sondern alle Berufsgruppen betreffen kann [1]. Entsprechend der International Labour Organisation (ILO) und der Europäischen Union ist Gewalt am Arbeitsplatz „,jede Handlung, Begebenheit oder von angemessenem Benehmen abweichendes Verhalten, wodurch eine Person im Verlauf oder in direkter Folge ihrer Arbeit schwer beleidigt, bedroht, verletzt oder verwundet wird“" $[2,3]$. Diese Definition von Gewalt betont den Bezug zum Setting Arbeitswelt. Die WHO definiert Gewalt als den „Gebrauch von angedrohtem oder tatsächlichem körperlichen Zwang oder psychischer Macht gegen die eigene oder eine andere Person, gegen eine Gruppe oder Gemeinschaft, der entweder konkret oder mit hoher Wahrscheinlichkeit zu Verletzungen, Tod, psychischen Schäden, Fehlentwicklungen oder Deprivation führt" [4]. Bei dieser Definition werden die Folgen von Gewalt differenziert beschrieben.

Diskriminierung definierte die ILO bereits im Jahr 1958 als jede Unterscheidung, Ausschließung oder Bevorzugung, die aufgrund der Rasse, der Hautfarbe, des Geschlechts, des Glaubensbekenntnisses, der politischen Meinung, der nationalen Abstammung oder der sozialen Herkunft vorgenommen werden und die dazu führen, die Gleichheit der Gelegenheiten oder der Behandlung in Beschäftigung oder Beruf aufzuheben oder zu beeinträchtigen [5]. In einer neueren Definition nennen die Arbeiterkammern in Österreich zusätzlich die sexuelle Orientierung und das Alter als Grund für Diskriminierungen:

Der Begriff Diskriminierung (aus dem Lateinischen für , unterscheiden', , aussondern') umfasst alle Äußerungen, Handlungen oder Unterlassungen, die Arbeitnehmerinnen und Arbeitnehmer insbesondere wegen

- ihres Alters,

- ihrer ethnischen Zugehörigkeit (z. B. Volksgruppe, Sprachgruppe, gemeint ist auch Hautfarbe),

- ihrer Religion,

- ihrer Weltanschauung,

- ihrer sexuellen Orientierung,

- ihres Geschlechts oder

- einer Behinderung

benachteiligen, verächtlich machen oder herabwürdigen. Auch Spott über eine andere Mentalität oder Lebensweise gilt als Diskriminierung. Diskriminierung entsteht oft durch Vorurteile z. B. älteren Arbeitnehmerinnen und Arbeitnehmern oder Migrantinnen und Migranten gegenüber. Vorurteile sind vorgefasste Einstellungen und Meinungen gegenüber bestimmten gesellschaftlichen Gruppen,

\begin{tabular}{|c|c|}
\hline \multicolumn{2}{|c|}{ Abkürzungen } \\
\hline$A G G$ & $\begin{array}{l}\text { Allgemeines Gleichbehandlungs- } \\
\text { gesetz }\end{array}$ \\
\hline ArbSchG & Arbeitsschutzgesetz \\
\hline$B G H W$ & $\begin{array}{l}\text { Berufsgenossenschaft Handel } \\
\text { und Warenlogistik }\end{array}$ \\
\hline$B G W$ & $\begin{array}{l}\text { Berufsgenossenschaft für Ge- } \\
\text { sundheitsdienst und Wohlfahrts- } \\
\text { pflege }\end{array}$ \\
\hline DGUV & $\begin{array}{l}\text { Deutsche Gesetzliche Unfallver- } \\
\text { sicherung }\end{array}$ \\
\hline GDA & $\begin{array}{l}\text { Gemeinsame Deutsche Arbeits- } \\
\text { schutzstrategie }\end{array}$ \\
\hline ILO & $\begin{array}{l}\text { International Labour Organisa- } \\
\text { tion }\end{array}$ \\
\hline LSV & $\begin{array}{l}\text { SpV Spitzenverband der land- } \\
\text { wirtschaftlichen Sozialversiche- } \\
\text { rung }\end{array}$ \\
\hline PTBS & $\begin{array}{l}\text { Posttraumatische Belastungsstö- } \\
\text { rung }\end{array}$ \\
\hline$R K I$ & Robert Koch-Institut \\
\hline TOP & $\begin{array}{l}\text { Technische, organisatorische und } \\
\text { personenbezogene Schutzmaß- } \\
\text { nahmen }\end{array}$ \\
\hline UV & Unfallversicherung \\
\hline$V B G$ & $\begin{array}{l}\text { Verwaltungs-Berufsgenossen- } \\
\text { schaft }\end{array}$ \\
\hline
\end{tabular}


die oft nicht auf eigene Erfahrungen zurückzuführen sind. Sie entstehen dadurch, dass Urteile, Ansichten oder Meinungen, die in unserer Gesellschaft vorhanden sind, übernommen und auf Einzelne übertragen werden, ohne ihren tatsächlichen Wahrheitsgehalt an der Realität zu überprüfen: „Ältere sind leistungsschwächer", lautet z. B. ein weitverbreitetes Vorurteil. Diese oft unbewusste Ausgrenzung und Benachteiligung aufgrund von Vorurteilen betrifft scheinbar nur eine Gruppe und nicht einzelne Personen. Verletzt fühlt sich aber jeder/jede Einzelne" [6].

Im vorliegenden Beitrag stellen wir die verschiedenen Formen von Gewalt und Diskriminierung am Arbeitsplatz und ihre Folgen dar. Ferner wird die Häufigkeit von Arbeitsunfällen, die durch Gewalt ausgelöst werden, untersucht. Das Arbeitsschutzgesetz und das Sozialgesetzbuch VII (gesetzliche Unfallversicherung) schaffen Voraussetzungen, um Gewalt und Diskriminierung am Arbeitsplatz zu verhüten und Opfer von Gewalt zu behandeln und zu rehabilitieren. Die Strategien zur Prävention und Rehabilitation werden im zweiten Teil des Aufsatzes beschrieben.

\section{Mobbing}

Unangemessenes, einschüchterndes, diskriminierendes oder verletzendes Verhalten kann situativ und singulär oder wiederholt und zielgerichtet gegen einzelne Personen oder Gruppen am Arbeitsplatz auftreten. Letzteres wird als Mobbing bezeichnet. Mobbing ist ein Verhalten von Vorgesetzten oder Kollegen, das systematisch und langfristig auf die Erniedrigung einer Person zielt. Mobbing wird gezielt eingesetzt, um eine Person zum Arbeitsplatzwechsel, in die Arbeitsunfähigkeit oder Kündigung zu drängen. Dementsprechend kann Mobbing sowohl zu psychischen als auch physischen Gesundheitsproblemen bei den Betroffenen führen [7-10].

Mobbing oder Bullying, wie es im Englischen bezeichnet wird, ist ein weitverbreitetes Problem [11]. Entsprechend einer norwegischen Erhebung sind 15\% der Beschäftigten davon betroffen [12]. Einer europaweiten Erhebung zufolge hatten $4 \%$ der abhängig beschäftigten Männer und 5\% der Frauen in den zwölf Monaten vor der Befragung Mobbing erlebt [13]. In einer deutschen, prospektiven Studie war der Gesundheitszustand von Mobbingopfern im Jahr vor Beginn des Mobbings schlechter als derjenige der Teilnehmer einer Vergleichsgruppe [14]. In einer norwegischen Studie waren psychische Probleme ein Risikofaktor, ein Opfer von Mobbing zu werden [15]. Ursache und Wirkung sind beim Mobbing nicht einfach zu trennen. Psychische Belastungen und Traumatisierungen können zu Verhaltensweisen führen, die das Risiko für Mobbing erhöhen. Mobbing kann dann zu weiterer Traumatisierung führen. Um den Kreislauf von psychischer Belastung und Mobbing zu durchbrechen, sind Interventionen zur Reduktion von psychosozialen Belastungen und aggressivem Verhalten am Arbeitsplatz notwendig. Mobbing erfüllt nicht die Voraussetzung, um als Arbeitsunfall anerkannt zu werden, da es sich um eine Einwirkung handelt, die nicht auf die Dauer einer Schicht begrenzt ist [16]. Zum Aufgabenspektrum der UV-Träger gehört allerdings die Verhütung arbeitsbedingter Gesundheitsgefahren, zu denen auch Mobbing zählt.

\section{Sexuelle Belästigung am Arbeitsplatz}

Eine besondere Form von Gewalt am Arbeitsplatz ist die sexuelle Belästigung. Darunter wird jedes vorsätzliche sexuell bestimmte Verhalten verstanden, das die Würde von Beschäftigten am Arbeitsplatz verletzt. Dazu gehören

- sexuelle Handlungen und Verhaltensweisen, die nach strafrechtlichen Regelungen unter Strafe gestellt sind, sowie

- sonstige sexuelle Handlungen und Aufforderungen zu diesen, sexuell bestimmte körperliche Berührungen, Bemerkungen sexuellen Inhalts und das Zeigen und sichtbare Anbringen von pornografischen Darstellungen, die von den Betroffenen erkennbar abgelehnt werden [17].

Die Besonderheit ergibt sich daraus, dass sexuelle Belästigung einseitig auf die Sexualität oder die sexualisierten Aspekte der Person oder Interaktion ausgerichtet ist. Belästigungen können nicht nur von Kollegen oder Vorgesetzten ausgehen, sondern auch von Kunden, Pflegebedürftigen oder deren Angehörigen. Eine aktuelle, im Auftrag der Antidiskriminierungsstelle des Bundes durchgeführte repräsentative Umfrage unter Beschäftigten in Deutschland ergab, dass $49 \%$ der befragten Frauen und 56\% der Männer schon einmal eine der im Allgemeinen Gleichbehandlungsgesetz (AGG) genannten Belästigungssituationen im Arbeitsumfeld erlebt haben. An der Spitze der Nennungen standen dabei „zweideutige Kommentare und Witze mit sexuellem Bezug“, die fast $40 \%$ der Frauen und fast $50 \%$ der Männer angaben. Nach eigenem Begriffsverständnis fühlten sich jede sechste Frau und jeder 14. Mann am Arbeitsplatz sexuell belästigt. Als Täter geben Frauen und Männer am häufigsten Männer an. Nur jede/r Fünfte weiß, dass ein Arbeitgeber seine Beschäftigten vor Belästigung schützen muss. Allerdings sind über $80 \%$ der Befragten der Meinung, dass Handlungsbedarf an bundesdeutschen Arbeitsplätzen bestehe [18]

In der Befragung wurden sexuelle Belästigungen „nach eigenem Begriffsverständnis“ seltener angegeben als diejenigen nach der Begriffsdefinition des AGG. Das ergibt sich aus der Problematik, dass sexuelle Belästigung schwer objektivierbar ist, weil unterschiedliche Personen die gleiche Belästigungssituation unterschiedlich bewerten können. Interessant ist vor diesem Hintergrund, dass die Mehrzahl der Befragten, Männer wie Frauen, Verhaltensweisen wie

- unerwünschtes Anstarren,

- anzügliche Bemerkungen,

- unerwünschte Berührungen und

- explizite Aufforderungen zu sexuellen Handlungen

als sexuell belästigend einstufte [18]. Es gibt also durchaus einen Konsens darüber, was im betrieblichen Miteinander zu unterlassen ist.

\section{Auswirkungen von sexueller Belästigung}

Die Auswirkungen von sexueller Belästigung können vielfältig sein. Insbesondere körperliche Übergriffe im Bereich der strafrechtlich relevanten Handlungen er- 
leben die Betroffenen als traumatisierend und sie können zu schweren gesundheitlichen Störungen führen. Aber auch verbale Belästigungen können nachhaltige Beeinträchtigungen zur Folge haben. Eine ältere Interviewstudie mit Betroffenen kommt zu folgendem Ergebnis: „Sexuelle Belästigungen werden als Verletzung der persönlichen Intimsphäre bewertet. Fast jede Gesprächspartnerin erinnerte sich an eine Belästigungssituation im Laufe ihres Berufslebens, die sie als besonders belastend und unangenehm erlebt hat und die für sie eine tief greifende Verletzung darstellt, unabhängig davon, ob es sich um verbale oder körperliche Über- oder Eingriffe in den Intimbereich gehandelt hat" [19, S. 92]. Für die Einschätzung einer belästigenden Situation als tief greifende Verletzung ist vor allem von Bedeutung, dass die Interviewten subjektiv keine Möglichkeit hatten, diesen Übergriff zu verhindern. Sie fühlten sich der Situation hilflos ausgeliefert. Einer Studie aus dem Jahr 1990 zufolge gaben die von sexueller Belästigung Betroffenen an, dass sie

- weniger Spaß am Beruf hätten,

- sich nicht mehr auf ihre Arbeit konzentrieren könnten,

- mit Krankheitssymptomen reagiert hätten,

- ihre Arbeitsstelle gekündigt hätten,

- Zweifel an ihrer beruflichen Eignung hätten,

- ihren erlernten Beruf oder ihre Karriereabsichten aufgegeben hätten [20, S. 32].

Neben den persönlichen Folgen für die Betroffenen haben Mängel in der Prävention von sexueller Belästigung auch für die Unternehmen negative Folgen: beispielsweise Gerichtsprozesse aufgrund von Klagen Betroffener, Einbußen in der Attraktivität als Arbeitgeber, ein schlechtes Betriebsklima mit Folgen für Gesundheit und Leistungsfähigkeit der Beschäftigten. Es gibt also viele gute Gründe, das Thema im Unternehmen nicht totzuschweigen, sondern offen und systematisch zu behandeln.

Neuere internationale Studien bestätigen diese negativen Auswirkungen von sexuellen Belästigungen am Arbeitsplatz auf das Wohlbefinden und die Gesundheit der Betroffenen [21]. Entspre-

Bundesgesundheitsbl 2016 · 59:88-97 DOI 10.1007/s00103-015-2263-x

(c) Springer-Verlag Berlin Heidelberg 2015

\section{A. Nienhaus · C. Drechsel-Schlund · H. Schambortski · A. Schablon}

Gewalt und Diskriminierung am Arbeitsplatz. Gesundheitliche Folgen und settingbezogene Ansätze zur Prävention und Rehabilitation

\section{Zusammenfassung}

Gewalt am Arbeitsplatz ist ein weitverbreitetes Problem, das in sehr unterschiedlichen Formen auftritt. Entsprechend vielfältig sind auch die Folgen für die Betroffenen und die Unternehmen. Sexuelle Belästigung ist eine Sonderform der Gewalt am Arbeitsplatz. Gewalt kann von betriebsfremden (bei Überfällen auf Kassierer/-innen) oder betriebseigenen Personen (Kollegen, Patienten, Betreuten) ausgehen. Etwa 16.000 Arbeitsunfälle aufgrund von Gewalt, die zu einer längeren Arbeitsunfähigkeit geführt haben, werden den Trägern der gesetzlichen Unfallversicherung (UV-Träger) jährlich gemeldet, wobei es eine ansteigende Tendenz gibt. Einem Survey nach sind Beschäftigte im Gesundheitswesen und in der Wohlfahrtspflege besonders von Gewalt betroffen. Sowohl psychische als auch physische Gewaltereignisse können zu schwerwiegenden Folgen wie z. B. zu einer posttraumatischen Belastungsstörung (PTBS) führen. Zur Vermeidung von Gewalt sind technische, organisatorische und persönliche Schutzmaßnahmen notwendig. Der Einsatz von ausgebildeten Deeskalationstrainerinnen und -trainern in besonders betroffenen Bereichen kann hilfreich sein. Für Opfer von psychischer und physischer Gewalt am Arbeitsplatz bieten die UV-Träger ein spezielles Psychotherapeutenverfahren an und empfehlen die Ausbildung von Erstbetreuern.

Schlüsselwörter

Arbeitsunfall · Sexuelle Belästigung · Deeskalationstraining · Prävention . Rehabilitation

\section{Violence and discrimination in the workplace. The effects on health and setting-related approaches to prevention and rehabilitation}

\section{Abstract}

Violence in the workplace is a widespread problem that manifests itself in very different forms. The consequences for victims and companies are equally diverse. Sexual harassment is a special form of violence at the workplace. Violence may come from external perpetrators (attacks on cashiers) or from persons inside a company or establishment (colleagues, patients, people in care). Statutory accident insurance institutions in Germany (UV, 'Unfallversicherungsträger") receive approximately 16,000 occupational injury reports per year that resulted from violence and led to extended incapacity to work. The numbers are increasing steadily. Particularly affected by violence are people working in healthcare and social welfare. Both psychological and physical violence can lead to severe disorders such as post-traumatic stress disorder (PTSD). To avoid violence, technical, organisational, and personal protective measures are needed. The training of de-escalation officers in the areas affected can be helpful. For victims of psychological and physical violence in the workplace, the UV offers special psychotherapeutic support and recommends the training of first-aiders.

Keywords

Occupational injury - Sexual harassment . De-escalation training . Prevention . Rehabilitation chend der bereits oben erwähnten europäischen Erhebung waren 3\% der abhängig beschäftigten Frauen und 1\% der Männer in den zwölf Monaten vor der Befragung von sexuellen Übergriffen betroffen. Eine amerikanische Studie zeigte, dass nicht nur psychosozial belastende Arbeitsbedingungen allgemein, sondern auch speziell sexuelle Belästigungen das Risiko für Arbeitsunfälle und arbeitsbedingte Erkrankungen erhöhen [22]. Ent- sprechend der Gesundheitsberichterstattung des Bundes kann das Erlebnis sexueller Gewalt zusätzlich zu den unmittelbaren negativen Folgen zu langfristigen körperlichen und psychischen Beschwerden, zu einem riskanten Bewältigungsverhalten (erhöhter Alkohol- und Nikotinkonsum) sowie zu vermehrten Komplikationen bei der Schwangerschaft oder Geburt und zu vermehrten Unterleibsoperationen führen [23]. 


\begin{tabular}{|c|c|c|c|c|c|}
\hline & \multicolumn{4}{|c|}{ Aggression und Gewalt } & \multirow[t]{2}{*}{ Alle Unfälle } \\
\hline & Alle & Betriebsintern & Fremde Personen & Sonstige & \\
\hline & $N(\mathrm{~S} \%)$ & $\begin{array}{l}N(\mathrm{~S} \%) \\
{[\mathrm{R} \%]}\end{array}$ & $\begin{array}{l}N(\mathrm{~S} \%) \\
{[\mathrm{R} \%]}\end{array}$ & $\begin{array}{l}N \\
\text { [R \%] }\end{array}$ & $\begin{array}{l}N \\
\text { (R \%) }\end{array}$ \\
\hline Verwaltungs-BG & $\begin{array}{l}4770 \\
(28,6)\end{array}$ & $\begin{array}{l}432(11,7) \\
{[9,1]}\end{array}$ & $\begin{array}{l}1559(25,5) \\
{[32,7]}\end{array}$ & $\begin{array}{l}2779 \\
{[58,3]}\end{array}$ & $145.802[3,3]$ \\
\hline BG für Gesundheitsdienst und Wohlfahrtspflege & $\begin{array}{l}4030 \\
(24,1)\end{array}$ & $\begin{array}{l}2032(55,0) \\
{[50,4]}\end{array}$ & $\begin{array}{l}778(12,5) \\
{[19,3]}\end{array}$ & $\begin{array}{l}1220 \\
{[30,3]}\end{array}$ & $68.296[5,9]$ \\
\hline BG Handel und Warenlogistik & $\begin{array}{l}1284 \\
(7,7)\end{array}$ & $\begin{array}{l}60(1,6) \\
{[4,7]}\end{array}$ & $\begin{array}{l}1103(17,7) \\
{[85,9]}\end{array}$ & $\begin{array}{l}121 \\
{[9,4]}\end{array}$ & $104.893[1,2]$ \\
\hline Unfallversicherung Bund und Bahn & $\begin{array}{l}1206 \\
(7,2)\end{array}$ & $\begin{array}{l}362(9,8) \\
{[30,0]}\end{array}$ & $\begin{array}{l}790(12,7) \\
{[65,5]}\end{array}$ & $\begin{array}{l}54 \\
{[4,5]}\end{array}$ & - \\
\hline BG für Transport und Verkehrswirtschaft & $\begin{array}{l}875 \\
(5,2)\end{array}$ & $\begin{array}{l}30(0,8) \\
{[3,4]}\end{array}$ & $\begin{array}{l}771(12,4) \\
{[88,1]}\end{array}$ & $\begin{array}{l}74 \\
{[8,5]}\end{array}$ & $57.435[1,5]$ \\
\hline BG Nahrungsmittel und Gastgewerbe & $\begin{array}{l}785 \\
(4,7)\end{array}$ & $\begin{array}{l}177(4,8) \\
{[22,5]}\end{array}$ & $\begin{array}{l}528(8,5) \\
{[67,3]}\end{array}$ & $\begin{array}{l}80 \\
{[10,2]}\end{array}$ & $68.806[1,2]$ \\
\hline BG der Bauwirtschaft & $\begin{array}{l}525 \\
(3,1)\end{array}$ & $\begin{array}{l}153(4,1) \\
{[29,1]}\end{array}$ & $\begin{array}{l}62(1,0) \\
{[11,8]}\end{array}$ & $\begin{array}{l}310 \\
{[59,1]}\end{array}$ & $105.248[0,5]$ \\
\hline Übrige UV-Träger & $\begin{array}{l}3219 \\
(19,3)\end{array}$ & $\begin{array}{l}449(12,2) \\
{[14,0]}\end{array}$ & $\begin{array}{l}641(10,3) \\
{[19,9]}\end{array}$ & $\begin{array}{l}2129 \\
{[66,1]}\end{array}$ & $324.034[1,0]$ \\
\hline Gesamt & $16.694(100,0)$ & $3695(100)[22,1]$ & $6232(100)[37,3]$ & $\begin{array}{l}6767 \\
{[40,5]}\end{array}$ & $874.514[1,9]$ \\
\hline
\end{tabular}

\section{Gewalt und Aggression als Arbeitsunfälle}

Gewaltereignisse am Arbeitsplatz fallen in der gesetzlichen Unfallversicherung als Arbeitsunfälle unter den Versicherungsschutz nach $₫ 8$ Siebtes Buch Sozialgesetzbuch - Gesetzliche Unfallversicherung - (SGB VII). Gewalt und Aggression am Arbeitsplatz führen bei etwa 16.000 Beschäftigten im Jahr zu einer längeren Arbeitsunfähigkeit. Dabei scheint es eine Tendenz zur Zunahme solcher Unfälle zu geben. Im Jahr 2011 wurden den Trägern der gesetzlichen Unfallversicherung (UV-Träger) 15.916 solcher meldepflichtigen Unfälle (mehr als drei Tage arbeitsunfähig) gemeldet. Im Jahr 2013 waren es 16.694 (plus 5\%) [24]. Diese Unfälle ereignen sich oft am Arbeitsplatz (96\%) und nur selten auf Dienstwegen (4\%). Mehr als ein Drittel dieser Unfälle wird durch Überfälle und Bedrohungen durch betriebsfremde Personen ausgelöst (• Tab. 1). Dahinter verbergen sich Überfälle auf Kassierer im Supermarkt und an Tankstellen, auf Bankangestellte, Spielhallenbetreiber oder Taxifahrer, um nur einige Beispiele zu nennen. Etwa jeder fünfte meldepflichtige Arbeitsunfall aufgrund von Gewalt und Aggression wird durch betriebseigene Personen verursacht. Das sind Kollegen oder beispielsweise Kunden, Betreute, Patienten oder Schüler. Die übrigen Arbeitsunfälle durch Gewalt und Aggression sind entweder nicht näher klassifiziert (19\%) oder beruhen auf einem Schreck- oder Überraschungsmoment, der nicht in aggressiver Absicht herbeigeführt wurde.

In absoluten Zahlen werden Arbeitsunfälle durch Aggression und Gewalt am häufigsten bei der Verwaltungs-Berufsgenossenschaft (VBG) gemeldet. Unfälle bei Schaustellern, in Spielhallen oder Ausbildungsstätten stehen hier im Vordergrund (persönliche Mitteilung). Von einem Drittel dieser Unfälle ist bekannt, dass sie durch betriebsfremde Personen verursacht wurden. Hier spielen Überfälle eine große Rolle. An zweiter Stelle steht die Berufsgenossenschaft für Gesundheitsdienst und Wohlfahrtspflege (BGW). Bei der BGW überwiegen Unfälle, die durch betriebsinterne Personen provoziert wurden $(50,4 \%)$. Gleichzeitig betrifft auch etwa die Hälfte aller Unfälle durch aggressive oder gewalttätige betriebsinterne Personen, die den UV-Trägern gemeldet werden, die BGW. Vor- herrschend sind hier allerdings nicht Gewalt unter Kollegen, sondern Übergriffe von Betreuten, Patienten und Angehörigen (siehe unten). VBG und BGW zusammen sind für mehr als die Hälfte aller meldepflichtigen Arbeitsunfälle, die durch Aggression und Gewalt am Arbeitsplatz ausgelöst wurden, zuständig. Gleichzeitig ist bei ihnen der Anteil der durch Gewalt verursachten Unfälle an allen meldepflichtigen Unfällen am höchsten (VBG $3,3 \%$, BGW 5,9\%). In manchen Branchen liegt der Anteil dieser Unfälle sogar noch höher, z. B. in der stationären Altenpflege bei $16 \%$ und in Werkstätten für Menschen mit Behinderungen bei $11 \%$ [berechnet nach 25]. Den größten Anteil an aggressions- und gewaltbedingten Unfällen haben jedoch die Berufsgenossenschaft für Transport und Verkehrswirtschaft (BG Verkehr) $(88,1 \%)$, die Berufsgenossenschaft Handel und Warenlogistik (BGHW) $(85,9 \%)$, die Berufsgenossenschaft für Nahrungsmittel und Gastgewerbe $(67,3 \%)$ sowie die Unfallversicherung Bund und Bahn (UVB) $(65,5 \%)$ (• Tab. 1).

„Die Täter sind meist männlich, sie kommen kurz vor Ladenschluss und sind häufig bewaffnet. Ihre Beute: Geld aus 


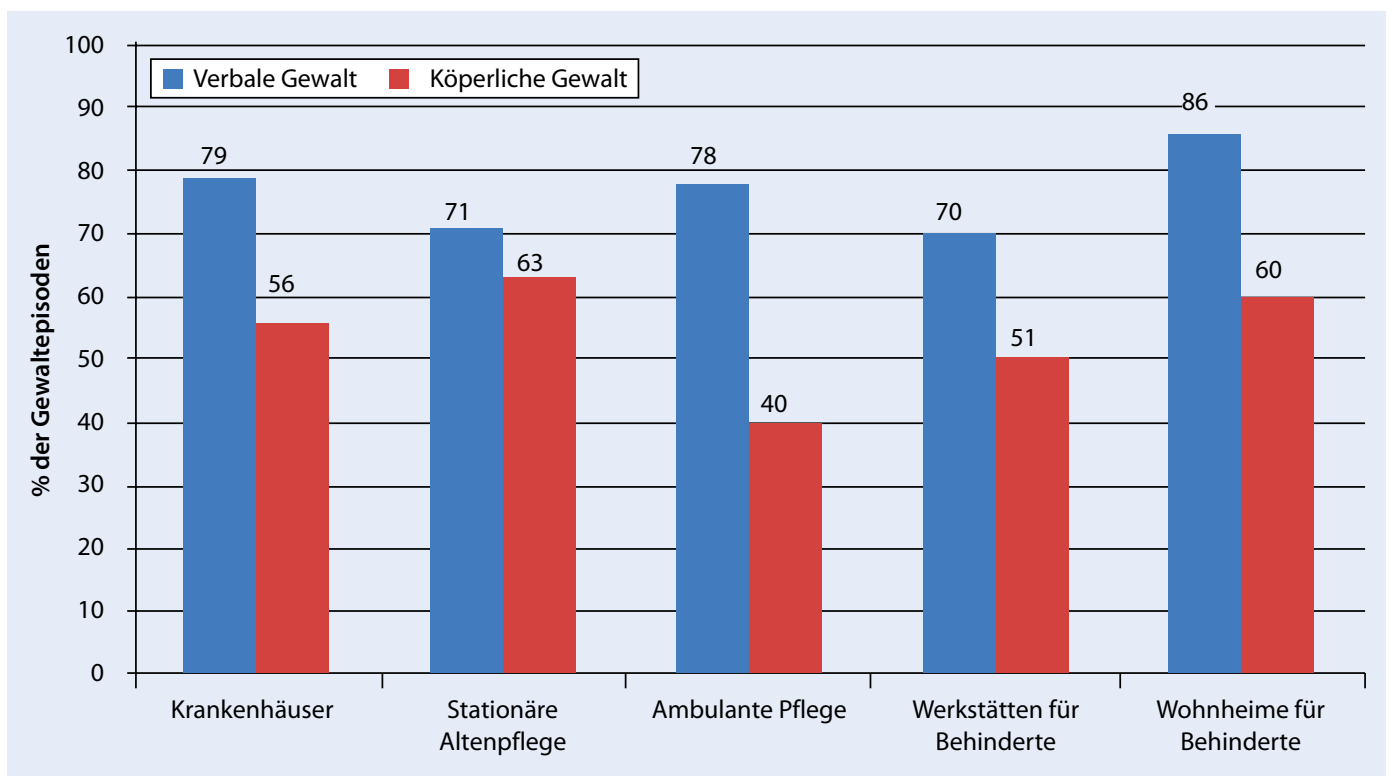

Abb. $1<$ Häufigkeit von verbaler und körperlicher Gewalt in den der Befragung vorangegangenen 12 Monaten, getrennt nach verschiedenen beruflichen Bereichen [29] den Ladenkassen oder Wertgegenstände. “ So beschreibt die BGHW die rund 1500 Raubüberfälle, die ihr gemeldet werden, weil die Opfer länger als drei Tage arbeitsunfähig sind [26]. Die Opfer sind meist weiblich, ist zu ergänzen. Die tatsächliche Anzahl der Raubüberfälle ist aber deutlich höher als die Anzahl der gemeldeten, weil die Opfer zwar psychisch belastet und traumatisiert werden, aber nicht immer arbeitsunfähig sind. Dann können die Überfälle zwar dennoch gemeldet werden und die UV-Träger können Hilfe anbieten (siehe unten), diese Unfälle sind aber nicht meldepflichtig nach dem Unfallversicherungsrecht. Sie werden deshalb in der Routinestatistik nicht als solche erfasst.

Die meldepflichtigen Arbeitsunfälle, die durch Gewalt am Arbeitsplatz ausgelöst werden, sind jedoch nur die Spitze des Eisberges. Tatsächlich sind Aggression und Gewalt am Arbeitsplatz viel häufiger. Die Erfassung dieser Ereignisse wird jedoch erschwert durch die vielfältigen Formen, in denen sie auftreten können. Die Angaben zur Häufigkeit dieser Übergriffe schwanken daher in der Literatur. In einer Übersichtsarbeit der verfügbaren internationalen Studien über Gewalt gegenüber Beschäftigten im Gesundheitsdienst gaben 11-96\% der Befragten an, in den vorangegangenen zwölf Monaten von Gewalt betroffen gewesen zu sein [27]. In einer deutschen Befragung von 1973 Beschäftigten aus verschiedenen Branchen des Gesundheitsdienstes und der Wohlfahrtspflege (außer Psychiatrie, siehe Aufsatz von Steinert und Traub in diesem Heft) erlebten $56 \%$ der Befragten körperliche und $78 \%$ verbale Gewalt in den der Befragung vorangegangenen zwölf Monaten $[28,29]$. Monatlich oder häufiger erlebten $44 \%$ der Befragten körperliche Gewalt und $68 \%$ verbale Gewalt. Die höchste Prävalenz körperlicher Gewalt wurde auf Altenpflegestationen (63\%) beobachtet, die geringste in der ambulanten Pflege $(40 \%)$. Beschäftigte in Wohnheimen für Menschen mit Behinderungen waren am häufigsten verbaler Gewalt ausgesetzt (86\%) (• Abb. 1). Neben verbaler Gewalt sind Drohgebärden häufig. Betroffen sind hier vor allem Beschäftigte in Einrichtungen für Menschen mit Behinderungen (Wohnheime $50 \%$, Werkstätten $42 \%$ ). Im Gegensatz dazu sind die Beschäftigten in der stationären Altenpflege (52\%) und in Krankenhäusern (42\%) am häufigsten Opfer von Kneifen und Kratzen. Beschäftigte in der stationären Altenpflege berichten häufiger über Schläge (35\%) als Befragte aus anderen Bereichen.

\section{Folgen von Gewalt}

Die Folgen von Gewalt am Arbeitsplatz können vielfältig sein. Allein im Bereich der BGW gibt es jährlich ein bis drei Todesfälle bei den Beschäftigten aufgrund dieser Unfälle. Bei den meisten Übergriffen stehen aber nicht körperliche, son- dern psychische Folgen im Vordergrund. Die Reaktionen können dabei sehr unterschiedlich sein. Eine reduzierte Arbeitszufriedenheit, Verunsicherung, Infragestellen der eigenen Person und Kompetenz, Gefühl der Ohnmacht, Motivationsverlust für die Arbeit, Erschöpfungsgefühle und Ängstlichkeit sind mögliche Reaktionsformen, die das Lebensgefühl und die Leistungsfähigkeit eines Beschäftigten langfristig beeinträchtigen können, ohne dass diese Übergriffe gemeldet und erfasst werden [30-34]. Die Betroffenen sind bei der Bewältigung dieser Übergriffe oftmals allein gelassen. Aber auch schwerwiegendere Reaktionen sind möglich. Depressive Verstimmungen, Schlafstörungen, Angstzustände, Vermeidungsverhalten und sozialer Rückzug können zu einer langfristigen Beeinträchtigung des Lebensgefühls, der Gesundheit und der Arbeitsfähigkeit führen. In einer Untersuchung von Opfern physischer Gewalt am Arbeitsplatz litt mehr als die Hälfte 30 Monate nach den Übergriffen noch unter psychischen Beeinträchtigungen [35].

Posttraumatische Belastungsstörungen entwickeln sich dabei häufig erst mit Verzögerung. Die Ausprägung der Störungen steht auch nicht in einem proportionalen Verhältnis zur Schwere des Übergriffs. Deshalb sind sie oftmals nicht leicht $\mathrm{zu}$ erkennen. Es ist daher wichtig die Schwere des Ereignisses, mögliche vorher bestehende Risikofaktoren (psychische Belastungen und Auffälligkeiten, wieder- 
Tab. 2 Das TOP-Prinzip bei der Prävention von Gewalt und Aggression im Betrieb

Präventionskultur, die Sicherheit und Gesundheit fördert

\begin{tabular}{|lll}
\hline Regelmäßige Durchführung von Gefährdungsbeurteilungen unter Einbeziehung der Beschäftigten & \\
\hline $\begin{array}{l}\text { Präventionsmaßnahmen auf der Ebene von tech- } \\
\text { nischen Lösungen }\end{array}$ & $\begin{array}{l}\text { Präventionsmaßnahmen auf der Ebene von orga-- } \\
\text { nisatorischen Lösungen }\end{array}$ & $\begin{array}{l}\text { Präventionsmaßnahmen auf der Ebene der be- } \\
\text { schäftigten Person }\end{array}$ \\
\hline Beispiele & Beispiele & Beispiele \\
\hline Bauliche Maßnahmen & Alarmierungsplanung & $\begin{array}{l}\text { Unterweisung über das Verhalten bei Gewalt- } \\
\text { vorfällen }\end{array}$ \\
\hline Sicherheitsglas & Rettungskette & $\begin{array}{l}\text { Schulung zu deeskalierendem Verhalten und ggf. } \\
\text { zu Befreiungs-/Selbstverteidigungstechniken }\end{array}$ \\
\hline Beleuchtung & $\begin{array}{l}\text { Handlungsspielräume der Beschäftigten zur De- } \\
\text { eskalation der Situation }\end{array}$ & $\begin{array}{l}\text { Angemessene Kleidung, Schuhwerk, Regeln zum } \\
\text { Tragen von Schmuck }\end{array}$ \\
\hline Fluchtwege und Rückzugsmöglichkeiten & $\begin{array}{l}\text { Strukturelle Gewalt identifizieren und hinter- } \\
\text { fragen }\end{array}$ & \\
\hline Alarmknöpfe & Fachkonzepte anpassen & \\
\hline Personennotsignalanlagen & $\begin{array}{l}\text { Systematische Auswertung von Vorfällen und } \\
\text { Beinahe-Vorfällen }\end{array}$ & \\
\hline $\begin{array}{l}\text { Eliminierung potenziell gefährlicher Gegen- } \\
\text { stände }\end{array}$ & & \\
\hline TOPTechnik, Organisation, Person. & & \\
\hline
\end{tabular}

holte Exposition) sowie die Reaktionen nach dem Ereignis (Leistungsabfall, Unkonzentriertheit, persönliche Probleme) zu beobachten, um frühzeitig einen Unterstützungs- oder Behandlungsbedarf zu erkennen [36]. Die soziale Unterstützung am Arbeitsplatz nach einem kritischen Ereignis scheint die Bewältigungsmöglichkeiten nach einem derartigen Trauma zu erhöhen [37]. Wenn die Einrichtung auf mögliche Übergriffe vorbereitet ist (siehe Prävention), reduziert sich das Belastungsempfinden aufgrund von Aggression und Gewalt am Arbeitsplatz [28, 29].

\section{Prävention von Gewalt und Aggression einschließlich sexueller Belästigung am Arbeitsplatz}

Wie immer im Arbeitsschutz orientieren sich die Präventionsmaßnahmen an der Gefährdungsbeurteilung entsprechend $\$ 5$ Abs. 3 Arbeitsschutzgesetz (ArbSchG). In ihr werden mögliche Gewaltsituationen analysiert und entsprechende Schutzmaßnahmen abgeleitet. Die Schutzmaßnahmen richten sich dabei nach dem TOP-Prinzip, wobei $\mathrm{T}$ für technische, $\mathrm{O}$ für organisatorische und $\mathrm{P}$ für persönliche Schutzmaßnahmen steht (• Tab. 2). Aggression, Gewalt oder sexuelle Belästigung können am besten vermieden werden, wenn die technischen, organisatorischen und auf die Person bezogenen Maßnahmen miteinander verzahnt sind.
Gewalt, Aggression, Mobbing oder sexuelle Belästigung gehören zu den psychischen Belastungen und sind deshalb auch Thema des Arbeitsprogramms Psyche 2013-2018 der Gemeinsamen Deutschen Arbeitsschutzstrategie (GDA) [38]. Unternehmen, in denen Gewaltvorfälle häufig auftreten, werden von den Mitarbeiterinnen und Mitarbeitern der Präventionsdienste der UV-Träger gezielt beraten und aufgefordert, das Thema „Gewalt“ in ihre Gefährdungsbeurteilungen zu integrieren.

Die Voraussetzung für das Gelingen präventiver Maßnahmen gegen Aggression und Gewalt am Arbeitsplatz ist eine betriebliche Kultur, die offen, systematisch und lösungsorientiert mit diesem Thema umgeht. Nur wenn das Thema „Aggression und Gewalt“ enttabuisiert wird, können die bewährten Präventionsmaßnahmen auf den Ebenen von Technik, Organisation und Person greifen [39]. Wenn Beschäftigte beispielsweise den Eindruck haben, von Vorgesetzten sowie Kollegen und Kolleginnen als inkompetent oder überempfindlich angesehen zu werden, wenn sie Gewalt- oder Belästigungsvorfälle melden, fehlt die Basis für das Präventionshandeln. Die UVTräger veröffentlichen deshalb regelmäßig Artikel zu diesem Thema in den Mitgliederzeitschriften und es finden Veranstaltungen und Vorträge statt. Es gibt mehr oder weniger umfangreiche Internetauftritte, die sich an Führungskräfte, Arbeits- schutzakteure und an Versicherte wenden. Beispiele dafür sind die Internetseiten der BGW (http://www.bgw-online.de/ gewalt) und der Unfallkassen (www.gesundheitsdienstportal.de/risiko-uebergriff). Aber auch andere UV-Träger bieten auf ihren Internetportalen entsprechende Informationen an.

\section{Verzahnung von Verhaltens- und Verhältnisprävention}

Zur Vermeidung der Eskalation einer aggressiven Situation ist die Qualifizierung des Personals in deeskalierender Kommunikation erforderlich. Die Fähigkeit, eine Situation einzuschätzen, zu wissen, wann klare Grenzen gesetzt werden müssen, wann Rückzug angemessen ist und wann durch deeskalierende Gesprächstechniken die Lage entschärft werden kann, bildet sich durch Erfahrungen, durch kollegiale Beratung und durch spezielle Trainings heraus. Entsprechend qualifizierte Fachkräfte sind besser in der Lage, schwierige Situationen zu meistern. Auszubildende und Berufseinsteiger, aber auch Hilfskräfte sind deshalb stärker gefährdet und müssen besonders unterwiesen und unterstützt werden. Entsprechende Qualifizierungsprogramme stehen mittlerweile in vielfältiger Form zur Verfügung.

Einige UV-Träger unterstützen ihre Unternehmen bei der Ausbildung von Deeskalationstrainern. Diese sind Kolle- 
Tab. 3 Auswahl von Angeboten der Berufsgenossenschaft für Gesundheitsdienst und Wohlfahrtspflege (BGW) zur Prävention von Gewalt am Arbeitsplatz und zur Rehabilitation nach diesen Ereignissen (Quelle: BGWintern [44])

\begin{tabular}{|lll}
\hline Ziel & Schrift, Flyer & Seminar/Gespräch, Beratung etc. \\
\hline Prävention & Konfliktmanagement und Mobbingprävention & Professioneller Umgang mit Gewalt und Aggression für Führungskräfte \\
\hline & Gewalt und Aggression in Betreuungsberufen & Betriebliches Konfliktmanagement und Prävention von Mobbing \\
\hline & Konflikte lösen, Arbeitsschutz stärken \\
\hline & Ausbildung innerbetrieblicher Deeskalationstrainerinnen und -trainer \\
\hline & & $\begin{array}{l}\text { Beratung beispielsweise zur Durchführung von Gefährdungsbeurteilungen oder } \\
\text { zum Aufbau eines systematischen Managements von Sicherheit und Gesundheit } \\
\text { bei der Arbeit }\end{array}$ \\
\hline Rehabilitation & Hilfe nach Extremerlebnissen & Probatorische Sitzungen nach Arbeitsunfällen und Berufskrankheiten \\
\hline & & Psychotherapie nach Arbeitsunfällen und Berufskrankheiten
\end{tabular}

gen und Kolleginnen, die als Multiplikatoren den betrieblichen Umgang mit Gewalt und Aggression organisieren und Schulungen zum Verhalten in kritischen Situationen in ihrem Arbeitsbereich durchführen. Mittlerweile gibt es mehrere Anbieter für solche Ausbildungen mit unterschiedlichen Konzepten in Bezug auf Inhalt und Zeitaufwand [39, S. 367]. Die Wirksamkeit verschiedener Ansätze zum Management von Aggression und Gewalt im Gesundheitswesen ist in der Literatur belegt [40-42]. Die Überprüfung der Wirksamkeit spezifischer Ansätze in verschiedenen Settings steht jedoch noch aus.

Die Bedeutung des Verhaltens der Beschäftigten zur Vermeidung von Gewaltereignissen darf nicht dazu führen, die Verantwortung für die betriebliche Prävention auf diese zu verlagern und technische oder organisatorische Lösungen aus dem Blick zu verlieren. Diese stellen auch weiterhin die Basis im Sinne der Verhältnisprävention dar, ohne die eine nachhaltige betriebliche Prävention nicht gelingen kann. Notsignalanlagen in Kombination mit einem Plan für das Notfallmanagement und ausgebildete Erstbetreuer für die Unterstützung von Mitarbeitern bei belastenden Ereignissen sind hier von Bedeutung [43]. Gerade die Sicherheit, die von guten technischen und organisatorischen Lösungen ausgeht, kann die Beschäftigten befähigen, sich in schwierigen Situationen angemessen zu verhalten. Das Wissen um Rückendeckung durch Kollegen und Vorgesetzte wirkt sich positiv auf das professionelle Verhalten der Beschäftigten aus. Die verschiedenen Präventionsebenen wirken zusammen und bedingen sich gegenseitig. So kann beispielsweise die Rettungskette nur funktionie- ren, wenn alle Beschäftigten unterwiesen wurden, wann und wie sie diese auslösen.

Gewalt und Aggression treten typischerweise in Arbeitsbereichen auf, in denen es Kontakte mit Kunden gibt. Bereiche, in denen strukturelle Vorgaben von den Beschäftigten umgesetzt werden müssen oder die Einhaltung von Regeln kontrolliert wird, sind besonders betroffen. Es ist außerdem ein Unterschied, ob Gewaltsituationen in kurzen, nicht auf langfristige Beziehungen angelegten Kontakten zu Kunden oder Klienten auftreten oder in länger dauernden Beziehungen zu Klienten, Patienten, Bewohnern oder Schülern. Im letztgenannten Fall sollten, sofern es sich nicht um schwere, strafrechtlich relevante Übergriffe handelt, deeskalierende und zum Selbstschutz eingesetzte Techniken gewährleisten, dass die Beziehung zu der Person, von der Gewalt ausgeht, nicht gefährdet wird. Beschäftigte müssen beim Einsatz von Deeskalations- oder Selbstschutztechniken die persönlichen Voraussetzungen der angreifenden Person mit ihren Erkrankungen oder Beeinträchtigungen berücksichtigen. Von daher ist es erforderlich, dass auf die unterschiedlichen Branchen zugeschnittene Präventionsmaßnahmen entwickelt werden und die Betriebe die Möglichkeit haben, diese entsprechend ihrer Besonderheiten zu gestalten. Die UV-Träger halten deshalb ein breites Angebot an branchenspezifischen Schriften, Seminaren und Beratungen vor, die beispielsweise Taxifahrer, Flugbegleiter, Wachpersonal, Lehrer, Pflege- und Betreuungskräfte oder Beschäftigte in Spielhallen in ihrer besonderen Situation ansprechen. Starre allgemeine Vorschriften sind nicht hilfreich. Empfehlungen sollten den Betrie- ben im Rahmen der Gefährdungsbeurteilung Raum geben, diese für ihre speziellen Gewaltsituationen und ihre spezielle Klientel zu modifizieren. In $\bullet$ Tab. 3 sind beispielsweise die speziellen Angebote der BGW zur Unterstützung der Betriebe bei der Prävention von Gewalt und Aggression wiedergegeben.

Wichtige Hinweise für die Prävention von sexueller Belästigung am Arbeitsplatz gibt die Antidiskriminierungsstelle [45]. Der Arbeitgeber muss alle Mitarbeiterinnen und Mitarbeiter über ihre Rechte bei Diskriminierung aufklären und betroffene Beschäftigte schützen. Wenn es sich bei den Tätern um Beschäftigte des Unternehmens handelt, können diese abgemahnt, umgesetzt und gekündigt werden. Bereits die Aufklärung wird unter den Beschäftigten die Sensibilisierung für korrektes Verhalten erhöhen. Die Einrichtung von betrieblichen Beschwerdestellen hilft Betroffenen, sich gegen Belästigungen zu wehren. Wichtige weitere Hilfestellung können die Gleichstellungsbeauftragen und die Betriebs- oder Personalräte sowie andere Personalvertretungen bieten. Gegen sexuelle Belästigungen durch Kunden, Patienten oder Klienten helfen Hausordnungen mit entsprechenden Verhaltensregeln, die den Beschäftigten Handlungsoptionen gegenüber den Belästigern bis hin zum Hausverbot oder der Kündigung des Dienstleistungsvertrags ermöglichen. Spezielle Selbstbehauptungstrainings insbesondere für Berufsanfänger können deren Fähigkeit zum Umgang mit Belästigungssituationen verbessern. Von entscheidender Bedeutung sind Führungskräfte, die für ein Betriebsklima sorgen, in dem über Belästigungen nicht gewitzelt wird, son- 


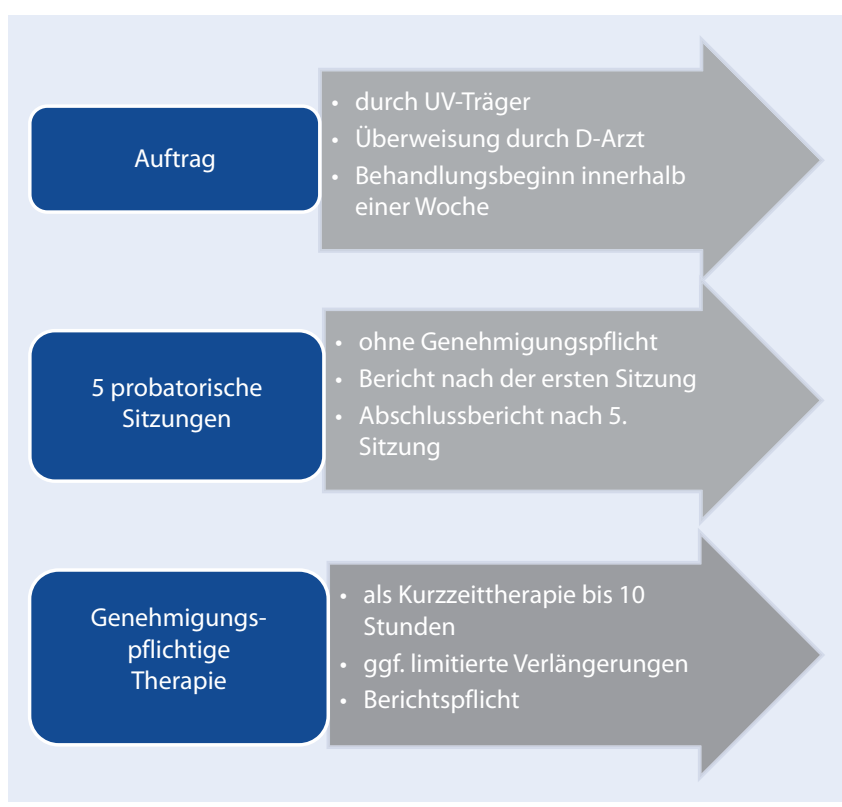

Abb. $2 \triangleleft$ Ablauf des Psychotherapeutenverfahrens. UV Unfallversicherung

dern in dem ein ernsthafter Erfahrungsaustausch und Unterstützung für die Betroffenen möglich sind.

Gewalt, Aggression und sexuelle Belästigung durch Patienten oder Bewohner mit demenziellen Erkrankungen sind eine besondere Herausforderung für die Beschäftigten. Mangelnde Affektkontrolle ist hier die Ursache für unangemessenes Verhalten. Spezielle Pflegekonzepte, wie beispielsweise Validation, sorgen für ein wertschätzendes Umfeld für die Betroffenen, das auch der Prävention von aggressiven Verhaltensweisen dient [46].

\section{Versorgung und Rehabilitation von Versicherten nach Gewaltereignissen}

Auch bei Übergriffen mit hohem psychischen Traumatisierungspotenzial, wie sie Gewaltereignisse darstellen, treten, sofern es sich um singuläre Ereignisse handelt, oftmals keine anhaltenden gesundheitlichen Folgen auf [47]. Bei einigen Personen entwickeln sich psychische Symptome, die eine frühzeitige psychotherapeutische Versorgung erfordern, um eine Chronifizierung zu verhindern [48]. Diese Fälle gilt es frühzeitig zu identifizieren. Zum Spektrum der psychischen Störungen nach Arbeitsunfällen, vor allem bei Gewaltereignissen, zählen die akute Belastungsreaktion, spezifische Phobien, PTBS,
Angststörungen, Anpassungsstörungen und depressive Episoden sowie anhaltende Schmerzstörungen in Verbindung mit körperlichen und psychischen Faktoren, die in kritischer Interaktion stehen können [49].

Mit dem am 1.7.2012 in Kraft getretenen Psychotherapeutenverfahren [50] stellen die UV-Träger sicher, dass die Diagnostik und Therapie von psychischen Störungen nach Arbeitsunfällen einschließlich Gewaltereignissen evidenzbasiert und leitliniengerecht erfolgt. Da die für Psychotherapie in der gesetzlichen Krankenversicherung geltenden Richtlinien nicht anwendbar sind, mussten spezifische Qualifikationsanforderungen und eigenständige Versorgungsstrukturen entwickelt werden. Durch ein Netz von bundesweit 473 (Stand 31.12. 2013) speziell für die Anforderungen der UV-Träger qualifizierten Psychotherapeuten wird eine zügige ambulante Versorgung und Rehabilitation von der Akutintervention bis zur beruflichen Reintegration gewährleistet [51]. Die UV-Träger und die Durchgangsärzte (Unfallchirurgen), denen Versicherte auch nach ggf. isolierter psychischer Gewalteinwirkung am Arbeitsplatz vorgestellt werden, fungieren als Lotsen bei der Heilverfahrenssteuerung, um den Zugang zum Psychotherapeutenverfahren mit probatorischen Sitzungen zu ermöglichen. Je nach Behandlungsbedarf kann sich auf Antrag eine Weiterbehandlung anschließen (• Abb. 2). Einzelne UV-Träger haben für Gewaltereignisse - ergänzend zum Psychotherapeutenverfahren spezielle Regelungen über Meldeverfahren und die Einschaltung von betriebsärztlichen sowie anderen psychologischen Diensten getroffen, um eine unmittelbare Betreuung bzw. Frühintervention bei den betroffenen Beschäftigten sicherzustellen [52]. Eine systematische Evaluation dieser Verfahren steht allerdings noch aus.

In das Versorgungsnetzwerk der UVTräger sind zudem die psychologischen Dienste der berufsgenossenschaftlichen Kliniken eingebunden [53, 54]. Das Leistungsspektrum umfasst spezielle berufsorientierte Trainings nach Gewaltereignissen am Arbeitsplatz, in denen Maßnahmen der medizinischen und beruflichen Rehabilitation verknüpft sind. Bei schweren Gesundheitsstörungen nach Gewaltereignissen kann eine Steuerung im RehaManagement der UV-Träger [55] geboten sein, um die medizinische Rehabilitation mit Versicherten, behandelnden Ärzten, Therapeuten und allen weiteren Beteiligten auf der Grundlage eines gemeinsam erstellten Rehabilitationsplans zu koordinieren und die Rückkehr an den Arbeitsplatz vorzubereiten.

Im Falle von Arbeitsunfällen durch Gewalt, Übergriffe und Aggression empfiehlt es sich, von Anfang an die Präventionsdienste der UV-Träger einzubinden. Ziel ist es zu klären, ob die Arbeitsschutzmaßnahmen ausreichend sind, um Wiederholungen vorzubeugen. Die Präventionsdienste sollten notwendige Optimierungen auf Grundlage einer Gefährdungsbeurteilung (siehe oben) frühzeitig anstoßen. Technische, organisatorische oder persönliche Schutzmaßnahmen sind möglichst vor Beginn der Wiedereingliederung des Betroffenen umzusetzen, um gemeinsam mit dem Arbeitgeber und den betrieblichen Arbeitsschutzakteuren die Voraussetzungen für eine schnelle und erfolgreiche Reintegration zu schaffen. Das Zusammenwirken der Bereiche Prävention und Rehabilitation der UV-Träger [56] ist für einen wirksamen Arbeits- und Gesundheitsschutz bei Gewalt, Übergriffen und Aggression am Arbeitsplatz von wesentlicher Bedeutung. 


\section{Korrespondenzadresse}

\section{Prof. Dr. A. Nienhaus}

Abteilung Grundlagen der Prävention und Rehabilitation

Berufsgenossenschaft für Gesundheitsdienst und Wohlfahrtspflege (BGW), Hamburg a.nienhaus@uke.de

\section{Einhaltung ethischer Richtlinien}

Interessenkonflikt. A. Nienhaus, C. DrechselSchlund, H. Schambortski und A. Schablon geben an, dass kein Interessenkonflikt besteht.

Dieser Beitrag beinhaltet keine Studien an Menschen oder Tieren.

\section{Literatur}

1. Chappell D, di Martino V (2006) Violence at work. Interational Labour Office, Geneva

2. Interational Labour Office (2009) Framework guidelines for addressing workplace violence in the health sector. International Labour Office 2002, Geneva

3. Europäische Agentur für Sicherheit und Gesundheitsschutz am Arbeitsplatz (2002) Gewalt bei der Arbeit. Facts 24. https://osha.europa.eu/de/toolsand-publications/publications?f[0]=field_p

4. di Martino V (2003) Workplace violence in the health sector-relationship between work stress and workplace violence in the health sector. ILO, ICN, WHO, PSI, Geneva

5. ILO (1960) Übereinkommen 111 Übereinkommen über die Diskriminierung in Beschäftigung und Beruf, 1958. http://www.ilo.org/wcmsp5/groups/public/-ed_norm/-normes/documents/normativeinstrument/wcms_c111_de.htm

6. Portal der Arbeiterkammern in Österreich (o J) Antidiskriminierung im Betrieb. Ein Ratgeber zum Erkennen und Bekämpfen von Diskriminierung im Betrieb. http://media.arbeiterkammer.at/wien/ PDF/Publikationen/ArbeitundRecht/Antidiskriminierung im Betrieb.pdf

7. Okechukwu CA, Souza K, Davis KD, de Castro AB (2014) Discrimination, harassment, abuse, and bullying in the workplace: contribution of workplace injustice to occupational health disparities. Am J Ind Med 57(5):573-586

8. Yang LQ, Caughlin DE, Gazica MW, Truxillo DM, Spector PE (2014) Workplace mistreatment climate and potential employee and organizational outcomes: a meta-analytic review from the target's perspective. J Occup Health Psychol 19(3):315-325

9. Nielsen MB, Magerøy N, Gjerstad J, Einarsen $S$ (2014) Workplace bullying and subsequent health problems. Tidsskr Nor Laegeforen 134(1213):1233-1238. (Artikel in Englisch und Norwegisch)

10. Loerbroks A, Weigl M, Li J, Glaser J, Degen C, Angerer $P$ (2015) Workplace bullying and depressive symptoms: a prospective study among junior physicians in Germany. J Psychosom Res 78(2):168172

11. Srabstein J, Joshi P, Due P et al (2008) Prevention of public health risks linked to bullying: a need for a whole community approach. Int J Adolesc Med Health 20(2):185-199
12. Nielsen MB, Skogstad A, Matthiesen SB et al (2009) Prevalence of workplace bullying in Norway: comparisons across time and estimation methods. Eur JWork Organ Psychol 18:81-101

13. Slany C, Schütte S, Chastang JF, Parent-Thirion A, Vermeylen G, Niedhammer I (2014) Psychosocial work factors and long sickness absence in Europe. Int J Occup Environ Health 20(1):16-25

14. Kostev K, Rex J, Waehlert L, Hog D, Heilmaier C (2014) Risk of psychiatric and neurological diseases in patients with workplace mobbing experience in Germany: a retrospective database analysis. Ger Med Sci 12:Doc10

15. Nielsen MB, Hetland J, Matthiesen SB, Einarsen S (2012) Longitudinal relationships between workplace bullying and psychological distress. Scand J Work Environ Health 38(1):38-46

16. Landessozialgericht Mecklenburg-Vorpommern vom 19.03.2014 Aktenzeichen: L 5 U 12/12

17. Allgemeines Gleichbehandlungsgesetz AGG $\S 3$ Begriffsbestimmung. http://www.gesetze-iminternet.de/agg/_3.html. Zugegriffen: 23. June 2015

18. Antidiskriminierungsstelle (2015) http://www. antidiskriminierungsstelle.de/SharedDocs/Downloads/DE/publikationen/Umfragen/Umfrage_sex_ Belaestigung_Los_1.html

19. Brandstedt U, Elke G, Schambortski H (1992) Sexuelle Belästigung am Arbeitsplatz: Wahrnehmungen und Bewältigungsstrategien berufstätiger Frauen - eine Studie. Frauenforschung 10(1/2):84104

20. Holzbecher M, Braszeit A, Müller U, Plogstedt S (1991) Sexuelle Belästigung am Arbeitsplatz. Schriftenreihe des Bundesministers für Jugend, Familie, Frauen und Gesundheit, 260. Kohlhammer, Stuttgart

21. Nielsen MB, Einarsen S (2012) Prospective relationships between workplace sexual harassment and psychological distress. Occup Med (Lond) 62(3):226-228

22. Brown LP, Rospenda KM, Sokas RK, Conroy L, Freels S, Swanson NG (2011) Evaluating the association of workplace psychosocial stressors with occupational injury, illness, and assault. J Occup Environ Hyg 8(1):31-37

23. Robert Koch-Institut (Hrsg) (2008) Gesundheitliche Folgen von Gewalt unter besonderer Berücksichtigung von häuslicher Gewalt gegen Frauen, Heft 42. Gesundheitsberichterstattung des Bundes, Berlin

24. DGUV (2015) Auskunft von Standke W, DGUV Referat,, Statistik - Makrodaten, Arbeits- und Schülerunfälle" vom 14.01.2015

25. Wendeler D, Dulon M, Nienhaus A (2014) Unfälle und Berufskrankheiten im Jahr 2012 bei der Berufsgenossenschaft für Gesundheitsdienst und Wohlfahrtspflege, In: Nienhaus A (Hrsg) RiRe -Risiken und Ressourcen in Gesundheitsdienst und Wohlfahrtspflege. ecomed, Landsberg

26. Berufsgenossenschaft Handel und Warendistribution (2015) BGHW aktuell April S.6

27. Zeh A, Schablon A, Wohlert C, Richter D, Niehaus A (2009) Gewalt und Aggression in Pflege- und Betreuungsberufen - ein Literaturüberblick. Gesundheitswesen 71:449-459

28. Schablon A, Zeh A, Wendeler D, Peters C, Wohlert C, Harling M, Nienhaus A (2012) Frequency and consequences of violence and aggression towards employees in the German healthcare and welfare system: a cross-sectional study. BMJ Open 2(5):1-5
29. Schablon A, Zeh A, Wendeler D, Wohlert C, Harling M, Nienhaus A (2014) Häufigkeit und Folgen von Gewalt und Aggression gegen Beschäftigte im deutschen Gesundheitswesen - ein Survey (deutschsprachige Variante von [28]. In: Nienhaus A (Hrsg) RiRe -Risiken und Ressourcen in Gesundheitsdienst und Wohlfahrtspflege. ecomed, Landsberg

30. Bernaldo-De-Quirós M, Piccini AT, Gómez MM, Cerdeira JC (2015) Psychological consequences of aggression in pre-hospital emergency care: cross sectional survey. Int J Nurs Stud 52(1):260-270

31. Edward KL, Ousey K, Warelow P, Lui S (2014) Nursing and aggression in the workplace: a systematic review. Br J Nurs 23(12):653-659

32. Demir D, Rodwell J (2012) Psychosocial antecedents and consequences of workplace aggression for hospital nurses. J Nurs Scholarsh 44(4):376-384

33. FitzGerald D, Reid A (2012) Frequency and consequences of violence in community pharmacies in Ireland. Occup Med (Lond) 62(8):632-637

34. Hartley D, Ridenour M, Craine J, Costa B (2012) Workplace violence prevention for healthcare workers - an online course. Rehabil Nurs 37(4):202206

35. De Puy J, Romain-Glassey N, Gut M, Wild P, Mangin $P$, Danuser B (2015) Clinically assessed consequences of workplace physical violence. Int Arch Occup Environ Health 88(2):213-224

36. McFarlane AC, Bryant RA (2007) Post-traumatic stress disorder in occupational settings: anticipating and managing the risk. Occup Med (Lond) 57(6):404-410

37. Regel S (2007) Post-trauma support in the workplace: the current status and practice of critical incident stress management (CISM) and psychological debriefing (PD) within organizations in the UK. Occup Med (Lond) 57(6):411-416

38. Gemeinsame Deutsche Arbeitsschutzstrategie GDA (o J) Arbeitsprogramm Psyche: Stress reduzieren - Potenziale entwickeln. http://www.gda-psyche.de/DE/Home/home_node.html

39. Walter G, Nau L, Oud N (Hrsg) (2012) Aggression und Aggressionsmanagement - Praxishandbuch für Gesundheits- und Sozialberufe. Verlag Huber, Bern

40. Richter D, Needham I (2007) Effekte von mitarbeiterbezogenen Trainingsprogrammen zum Aggressionsmanagement in Einrichtungen der Psychiatrie und Behindertenhilfe - Systematische Literaturübersicht. Psychiatr Prax 34:7-14

41. Livingston JD, Verdun-Jones S, Brink J, Lussier P, Nicholls T (2010) A narrative review of the effectiveness of aggression management training programs for psychiatric hospital staff. J Forensic Nurs $6: 15-28$

42. Kynoch K, Wu CJ, Chang AM (2011) Interventions for preventing and managing aggressive patients admitted to an acute hospital setting: a systematic review. Worldviews Evid Based Nurs 8:76-86

43. DGUV (2015) Gut vorbereitet für den Ernstfall -Mit traumatischen Ereignissen im Betrieb umgehen. DGUV Information 206-017. http://publikationen. dguv.de/dguv/pdf/10002/206-017.pdf

44. BGWintern Produktlinie Psyche und Gesundheit Stand 05/2015, Hamburg

45. Antidiskriminierungsstelle des Bundes (o J) Grenzen setzen - Was tun bei sexueller Belästigung am Arbeitsplatz? http://www.antidiskriminierungsstelle.de/SharedDocs/Downloads/DE/publikationen/Plakate/Plakat_Grenzen_setzen.pdf? blob=publicationFile 
46. Bundesministerium für Gesundheit (2006) Rahmenempfehlungen zum Umgang mit herausforderndem Verhalten bei Menschen mit Demenz in der stationären Altenpflege. https://www.bundesgesundheitsministerium.de/fileadmin/dateien/Publikationen/Pflege/Berichte/Bericht_Rahmenempfehlungen_zum_Umgang_mit_herausforderndem_Verhalten_bei_Menschen_mit_Demenz_in_der_stationaeren_Altenhilfe.pdf

47. Angenendt J (2012) Psychische Störungen nach Gewalterleben und Bedrohung - Einführung aus medizinisch-psychologischer Sicht. MedSach 108(3):106-110

48. Angenendt J, Riering A, Röhrich B, Südkamp N, Berger M (2012) Freiburger Arbeitsunfallstudie-II (FAUST-II) Trauma Berufskrankh 14(Suppl 3):299306

49. Bengel J (2014) Akute Folgen psychischer Traumatisierung - Diagnostik und Behandlung AWMFRegister Nr. 051/027. http://www.awmf.org/leitlinien/detail/anmeldung/1/ll/051-027.html

50. DGUV, LSV-SpV (2012) Psychotherapeutenverfahren. http://www.dguv.de/medien/landesverbaende/de/med_reha/documents/psych2.pdf

51. Scholtysik D (2015) DGUV-Referat Soziale Reha, Begutachtung, Pflege, Psyche. Persönliche Auskunft vom 6.2.2015

52. BGHW (o J) Hilfe nach einem Überfall/Gewaltereignis. http://www.bghw.de/arbeitnehmer/unsere-leistungen/hilfe-nach-einem-ueberfall-gewaltereignis/hilfe-nach-einem-ueberfall-gewaltereignis

53. Schulz B, Ullmann U (2007) Psychotraumatologische Versorgung im bg-lichen Heilverfahren. Trauma Berufskrankh 9(Suppl 1):109-112

54. Drechsel-Schlund C, Weiß M, Krahl C, Romer-Raschidi K, Gruner B, Freytag H, Hoffmann R (2015) Umsetzung des Psychotherapeutenverfahrens, Trauma und Berufskrankheit (online first). http:// link.springer.com/journal/10039/onlineFirst/page/2

55. DGUV (o J) Reha-Manager/Berufshelfer. http:// www.dguv.de/de/Rehabilitation-Leistungen/Berufliche-und-soziale-Teilhabe/Reha-Manager-Berufshelfer/index.jsp

56. Drechsel-Schlund C, Windemuth D (2013) Gewalt am Arbeitsplatz - Handlungsfelder für Prävention und Rehabilitation. DGUV Forum 7-8:18-19 